\title{
RISKS OF VACCINATION: A REVIEW
}

\section{Rashid A (1), Rasheed K (2), Asim M (1), Hussain A (1)}

(1) Division of Virology, Cell Culture Section, Veterinary Research Institute, Lahore Cantt, Pakistan; (2) University of Veterinary and Animal Sciences, Lahore, Pakistan.

\begin{abstract}
To be granted a product license, a vaccine must present adequate quality, safety and efficacy. Studies on these criteria often utilize target species in a laboratory setting. Vaccines that had been proven effective during laboratory analysis may not present the same features when sold on a large scale, after encountering field conditions, and furthermore, can even produce complications. Measures are already in place to detect adverse reactions as reported by veterinarians to manufacturers, so that vaccines under suspicion of failing can be identified and investigated. The present review article describes the main problems, specifically adverse reactions and lack of efficacy, that have been encountered following release of vaccines for general use.
\end{abstract}

KEY WORDS: vaccine, quality, safety, efficacy, adverse reactions.

CONFLICTS OF INTEREST: There is no conflict.

\section{CORRESPONDENCE TO:}

ASIF RASHID, Division of Virology, Cell Culture Section, Veterinary Research Institute, Ghazi Road, Lahore Cantt, Pakistan. Phone: +92 300 4033654. Email: dr asif rasheed@hotmail.com. 


\section{INTRODUCTION}

Safety is a concept that does not rest on well understood and generally accepted boundaries. Although the word "safety" may be explained as a state in which humans and other animals are uninjured, whole and healthy, the extension of this definition to the more abstract, universalized and future state of safety raises questions (1).

A vaccine may be considered unsafe when it causes disease or harm to a recipient or any individual associated with its production or delivery process. A vaccine that presents maximum safety is normally the product of a consistently well run process, the components of which have been thoroughly pretested before admittance into mainstream production.

Vaccination safety i.e., ensuring and monitoring the safety of all aspects of immunization, including vaccine quality, storage and handling, vaccine administration and disposal of sharps, remains a major challenge (2). Immunization can cause adverse events, ranging from inherent properties of vaccines to some error in the immunization process. The aim of effective vaccination is the generation of memory cells, namely memory $B$ and memory $T$ cells that are responsible for providing a fast and efficient immunity in a second contact with specific antigens $(3,4)$.

Vaccines, in contrast to other pharmaceutical products, are administered to healthy subjects because they aim to prevent, not to treat (5). Vaccines currently recommended for use in food animals and equine medicine and those given to pets consist mostly of dead bacteria and inactivated viruses, live attenuated bacteria and viruses, subunits of synthetic peptides, and toxoid vaccines produced by methods, or modifications of methods, that have been employed for decades (6).

Effective vaccines, developed for use in livestock, are expected to reduce the incidence and severity of diseases (7). However, reports of poor efficacy and unsightly reactions at the inoculation site have led to much confusion among livestock owners about the choice of vaccine (8).

Mild local and systemic reactions to vaccines are likely to be a natural consequence of vigorous stimulation of the immune system. Dramatic adverse responses are occasionally observed due to mistakes during production or handling of the products. More often, reactions are consequences of disobeying label instructions, particularly their restrictions against using vaccines only in healthy animals (9). It is important to publish well-documented instances of adverse vaccine reactions so that producers 
and users of these products can learn from previous experiences and avoid similar problems.

Animals affected by immunization side effects may show symptoms within a few minutes or, sometimes, after a delay of several hours. In the acute fulminating type of reaction, animals show labored breathing (dyspnea), sweating, incoordination, prostration, sporadic violence, severe colic symptoms, frothy blood-stained discharge from the mouth and nostrils, and sometimes anal discharge followed by death (10).

Some policies are already in place to detect adverse vaccine reactions through reports from veterinarians to manufacturers, so that products under suspicion of failure can be identified and investigated. The present study describes the two main problems that have been encountered subsequent to release of vaccines for general use; adverse reactions and lack of efficacy.

\section{ADVERSE REACTIONS}

Many types of adverse responses have been reported following vaccination, ranging from mild reaction (at the injection site) to more serious side effects (Table 1 ). Anaphylaxis and some autoimmune diseases such as immune-mediated hemolytic anemia are also adverse reactions to vaccination (11). One report clearly documents the elevation in humoral aspect but depression of cellular and innate immunity in a dog two weeks after immunization (12). However, post-vaccine immunosuppression is not a consistent observation among studies (13-15). Vaccination-induced immunosuppression may be sufficient to permit the development of severe diseases in animals that are already carrying subclinical opportunistic pathogens (16).

Table 1. Post-vaccination adverse reactions

\begin{tabular}{c|l}
\hline Class & Type of Reaction \\
\hline I & Not related to vaccine \\
\hline II & Lump or swelling at the injection site \\
\hline III & Facial swelling \\
\hline IV & Systemic signs, fever, vomiting, diarrhea \\
\hline V & Anaphylaxis, shock, collapse, death \\
\hline
\end{tabular}


Care must be taken to ensure that vaccines are not administered through an inappropriate route, either directly or indirectly. It has been reported that accidental intranasal administration of vaccines against feline respiratory diseases, which should be inoculated subcutaneously, may result in replication of viruses in the animal's oropharynx and other clinical diseases. Similarly, accidental infection of susceptible non-target hosts with vaccinal virus may lead to serious problems, for example, encephalitis in young dogs caused by canine distemper vaccine given to their dam in a booster dose when puppies were three days old (18).

Adverse reactions caused by incorrect procedures of storage, handling or administration are particularly interesting because they are more frequent than events associated with intrinsic properties of vaccines and can be easily resolved (Table 2). For instance, post-vaccinal distemper encephalitis is a rare condition that can occur after administration of a modified live-virus canine distemper vaccine. The affected animal may present aggression, incoordination and seizures or other neurological signs. The pathogenesis of this condition is unknown but may be due to residual virulence, increased susceptibility to, or triggering of, a latent paramyxovirus by the vaccine (19).

Table 2. Inappropriate procedures and associated adverse events

\begin{tabular}{l|l}
\hline Procedure & Potential adverse event \\
\hline Incorrect storage & Local reaction \\
\hline Incorrect injection site or technique & Local reaction \\
\hline Non-sterile procedures & Local reaction \\
\hline Contamination of vaccines or syringes & Toxic shock syndrome \\
\hline Contraindications ignored & Anaphylaxis \\
\hline Wrong diluents or substitution with another drug & Death \\
\hline
\end{tabular}

Adapted from: Albert ET (20).

The key to managing adverse events is communication. Anticipatory guidance should always be given to animal owners in the form of information on type and frequency of adverse events that could be experienced.

Anaphylaxis is a serious and potentially fatal hypersensitivity reaction that leads to a variety of distressing symptoms. Besides antigens, vaccines contain several other components including preservatives, adjuvants and manufacturing residues. In this 
context, it is not always clear which compound may be responsible for allergic reactions. For example, in some vaccines, gelatin and egg proteins are present at levels sufficient to induce hypersensitivity (21). However, the levels of mercury, aluminum, formaldehyde, albumin and antibiotics that are present in vaccines have not been shown to be harmful to humans or experimental animals (22).

Vaccination has been linked to a range of autoimmune disorders (particularly in dogs) including immune-mediated hemolytic anemia (IMHA), immune-mediated thrombocytopenia (IMTP), polyneuritis and polyarthritis $(11,23,24)$. There are few data that define the mechanism by which these associations may arise, although hypothetically a number of possibilities could be proposed, including disturbance of immunoregulation by vaccination; non-specific immune activation via adjuvants or microbe-derived "superantigen" exposed to cross-reactive tissue protein incorporated into vaccines; and molecular mimicry between autoantigens and vaccinal microbes (25).

Local injection-site reactions are rarely reported in pets, although it is clear that tissue inflammation does occur at vaccination sites, particularly on account of adjuvant products. The most visible of local reactions consist of the range of aggressive feline sarcoma in which adjuvant-induced chronic inflammation may trigger genetic changes that underlie neoplasia $(26,27)$.

In some cases, following vaccination, a lump appears at the inoculation site and may result in abscesses after infection enters through the pierced skin. The lumps are hard and fibrous, and arise when a vaccine that should be injected into deep intramuscular tissues leaks out into shallower subcutaneous tissues. In some animals, administration of alum-precipitated vaccine results in a local reaction that gradually leads to induration resulting in persistent hard lump (28).

Similarly, type IV or cell-mediated hypersensitivity reactions may occur in response to vaccination but a more common reaction is granuloma formation at the inoculation site. This may be a response to depot adjuvants that produce larger and more persistent lesions at inoculation sites than vaccines containing alum or aluminum hydroxide (19).

Other phenomena known as "physiological reactions" occur after vaccination. One example of these responses is the rise of 1.2 to $1.5 \%$ in rectal temperature in some animals 24 hours after vaccination against hemorrhagic septicemia; but the temperature usually returns to its normal value at 48 hours, except for buffaloes (in 
which the elevation persists slightly longer) (29). Analogous reactions were observed by Shah Najmul (30), studying the effects of single- $(2 \mathrm{~mL})$ and double-dose administration of oil adjuvant vaccine to five healthy buffalo calves aged from six to eight months, who found approximately a $2^{\circ} \mathrm{C}$ rise in body temperature at six hours after vaccination. The respiration rate and feed intake remained unaffected. Two calves showed a mild transient depression at six hours after vaccination.

\section{LACK OF EFFICACY}

Veterinary vaccines from different manufacturers can vary in their potency, efficacy and duration of immunity. Failure of vaccines to adequately protect from infectious disease is clearly a serious problem, but is more often due to inappropriate administration or administration to immunosuppressed or immunodeficient individuals, than to batches of subnormal efficacy (31). Moreover, it is important to remember that no current vaccine can claim $100 \%$ efficacy on the basis of experimental challenge or field studies. It has long been recognized that some dog breeds (such as Rottweiler and Doberman Pinscher) present increased susceptibility to parvoviral enteritis that may be related to suboptimal response to vaccination, although specific studies have not addressed the mechanism by which this might occur (32).

Efficacy can also be reduced by storage or reconstitution conditions; only small doses of live organisms are found in attenuated vaccines and these organisms may be killed by poor handling. Similarly, malnutrition can suppress immune response by decreasing availability of nutrients for cell division and protein synthesis.

A vaccine failure is relatively easy to detect when animals develop an acute infection, but is much less likely to be suspected when the infection is chronic or when the agent is absent from the community (18).

\section{CONCLUSIONS}

Certain factors including vaccine, immunized organism and environment influence the product efficacy. Vaccination risks may range from severe adverse responses to lack of efficacy. However, correct storage, improved inoculation technique and animal screening before immunization for concurrent infection may improve effectiveness by decreasing the incidence of adverse reactions. Finally, veterinarians 
should use only licensed vaccines and carefully follow manufacturer recommendations to avoid the risks.

\section{REFERENCES}

1. Spier RE. Ethical aspects of the methods used to evaluate the safety of vaccines. Vaccine. 2004;22(15-16):2085-95.

2. Duclos $P$, Hofmanin CA. Immunization safety. A priority of the World Health Organization's Department of Vaccines and Biologicals. Drugs Saf. 2001;24:1105-12.

3. Berek C, Ziegner M. The maturation of the immune response. Immunol Today. 1993;14(8):400-4.

4. Sander ME, Makgoba MW, Shaw S. Human naïve and memory $\mathrm{T}$ cell; reinterpretation of helper-induced and suppressor-induced subsets. Immunol Today. 1988;9(7-8):195-9.

5. Chen RT. Vaccine risks: real, perceived and unknown. Vaccine. 1999;17(3):S41-6.

6. Carter PB, Carmichael LE. Modern veterinary vaccines and Shaman's apprentice. Comp Immunol Microbiol Infect Dis. 2003; 26(5-6):389-400.

7. Smith LDS. Clostridial diseases of animals. In: Brandly CA, Jungherr EL, editors. Advances in Veterinary Science. New York: Academic Press; 1957.

8. Smith HV and Klose JB. Response of goats to clostridial vaccines. Victorian Veterinary Proceedings. 1980;38:46.

9. Roth JA. Mechanistic bases for adverse vaccine reactions and vaccine failures. Adv Vet Med. 1999;41:681-700.

10. De Alwis MCL. Haemorrhagic Septicaemia. Canberra: Australian Centre for International Agricultural Research; 1999. 141 p.

11. Duval D, Ginger U. Vaccine associated immune-mediated hemolytic anemia in the dog. J Vet Intern Med. 1996;10(5):290-5.

12. Strasser A, May B, Teltscher A, Wistrela E, Niedermuller H. Immune modulation following immunization with polyvalent vaccine in dogs. Vet Immunol Immunopathol. 2003;94(3-4):113-21.

13. Phillips TR, Jensen JL, Rubino MJ, Yang WC, Schultz RD. Effects of vaccines on the canine immune system. Can J Vet Res. 1989;53(2):154-60.

14. McMillen GL, Briggs DJ, McVey DS, Phillip RM, Jordon FR. Vaccination of racing greyhounds: effects on humoral and cellular immunity. Vet Immunol Immunopathol. 1995;49(1-2):101-13. 
15. Miyamoto T, Taura Y, Une S, Yoshitake M, Nakama S, Watanabe S. Changes in blastogenic responses of lymphocytes and delayed type hypersensitivity responses after vaccination in dogs. J Vet Med Sci. 1992;54(5):945-50.

16. Foley JE, Orgad U, Hirsh DC, Poland A, Pedersen NC. Outbreak of fatal salmonellosis in cats following use of a high-titer modified-live panleukopenia virus vaccine. J Am Vet Med Assoc. 1999;214(1):67-70, 43-4.

17. Novak W. Predicting the "unpredictable" vaccine reactions. Proceedings of North American Veterinary Conference. Ithaca: International Veterinary Information Service; 2007.

18. Ramsey I, Bryn T (editors). BSAVA Manual of canine and feline infectious diseases. [Place unknown]: BSAVA; 2001. 46-48 p.

19. Tizard IR. Veterinary Immunology: an Introduction. 6th ed. Philadelphia: W.B. Saunders Company; 2000. 239 p.

20. Tozzi AE. Field evaluations of vaccine safety. Vaccine. 2004;22(15-16):2091-5.

21. O' Hagen DT, Rino R. The safety of vaccines. Drug Discovery Today. 2004;9(19):846-54.

22. Offit PA, Jew RK. Addressing parents' concern: do vaccines contain harmful preservatives, adjuvants, additives or residues? Pediatrics. 2003;112(6):1394-7.

23. Kohn B, Garner M, Lubke S, Bennett D, Brunnberg L. Polyarthritis following vaccination in four dogs. Vet Comp Orthroped Traumatol. 2003;16:6-10.

24. McAnulty JF, Rudd RG. Thrombocytopenia associated with vaccination of a dog with a modified live paramyxovirus vaccine. $\mathrm{J}$ Am Vet Med Assoc. 1985;186(11):1217-9.

25. Schattner A. Consequence or coincidence? The occurrence, pathogenesis and significance of auto-immune manifestations after viral vaccines. Vaccine. 2005;23(30):3876-86.

26. McEntee MC, Page RL. Feline vaccine-associated sarcomas. J Vet Intern Med. 2001;15(3):176-82.

27. Richards JR, Starr RM, Childers HE, Elston TH, Hendrick MJ, Kitchell BE, Macy DW, McClure D, Morrison WB, Vogel LP, Welborn LV. Vaccine-associated feline sarcoma task force: roundtable discussion. J Am Hosp Assoc. 2005;226(11):182142.

28. Nagarajan V, Sunderam S. Neutralization of the Hemorrhagic Septicaemia alum precipitated vaccine and its effects on local reactions. Indian Vet J. 1975;52:800-2. 
29. Pandy MR, Singh ND, Yadev RS. Effect of vaccination against hemorrhagic septicaemia on milk yield, body temperature and feed intake of Therparker crossbred cow and Murrah buffaloes. A note. Indian J Anim Sci. 1987;3:35-6.

30. Shah Najmul H. Haemorrhagic septicaemia in dairy buffaloes in Pakistan: pathogenesis and prevention [PhD thesis]. Amsterdam: Vrije University of Amsterdam; 1998.

31. Day MJ. Vaccine side effects: fact and fiction. Vet Microbiol. 2006;117(1):51-8.

32. Hoskins JD. Performance of a new generation canine parvovirus vaccine in Rottweiler puppies. Can Pract. 1997;22(4):29-31. 